\title{
On quasimultiperfect numbers
}

\author{
P. Anantha Reddy ${ }^{1}$, C. Sunitha ${ }^{2}$ and V. Siva Rama Prasad ${ }^{3}$ \\ ${ }^{1}$ Q.Q. Govt. Polytechnic \\ Chandulalbaradari, Hyderabad, Telangana-500064, India \\ e-mail: ananth_palle@yahoo.co.in \\ 2 Department of Mathematics and Statistics, RBVRR Womens College \\ Narayanaguda, Hyderabad, Telangana-500027, India \\ e-mail: csunithareddy1974@gmail .com \\ ${ }^{3}$ Professor(Retired), Department of Mathematics, Osmania University \\ Hyderabad, Telangana-500007, India \\ e-mail: vangalasrp@yahoo.co.in
}

Received: 20 November 2019

Revised: 5 August 2020

Accepted: 5 August 2020

\begin{abstract}
For a positive integer $n$, let $\sigma(n)$ and $\omega(n)$ respectively denote the sum of the positive divisors of $n$ and the number of distinct prime factors of $n$. A positive integer $n$ is called a quasimultiperfect (QM) number if $\sigma(n)=k n+1$ for some integer $k \geq 2$. In this paper we give some necessary conditions to be satisfied by the prime factors of QM number $n$ with $\omega(n)=3$ and $\omega(n)=4$. Also we show that no QM $n$ with $\omega(n)=4$ can be a fourth power of an integer.
\end{abstract} Keywords: Quasimultiperfect number, Quasitriperfect number.

2010 Mathematics Subject Classification: 11A25.

\section{Introduction}

For a positive integer $n$, let $\sigma(n)$ and $\omega(n)$ respectively denote the sum of the positive divisors of $n$ and the number of distinct prime factors of $n$. Tang Min and Meng Li [4] called a positive integer $n$ quasimultiperfect (QM) number if $\sigma(n)=k n+1$ for some integer $k \geq 2$. In particular, a positive integer $n$ is said to be quasiperfect (QP) if $\sigma(n)=2 n+1$ and quasitriperfect (QT) if $\sigma(n)=3 n+1$. No QM number is known so far. P. Cattaneo [2] started the study of QP numbers which was continued in [1] and later by several researchers, the details of which can be seen in the book [7, p.38-39] and in recent papers [5] and [6]. 
If a QM number $n$ exists, then it is shown in [4, Theorem 1] that $\omega(n) \geq 7$ or 3 according as $n$ is odd or even. Also it is proved:

Lemma 1.1 ([4, Theorem 2]). If $n$ is an even $Q M$ with $\omega(n)=3$, then $n$ is $Q T$ and is of the form $n=2^{\alpha} \cdot 3^{\beta} \cdot p^{2}$, where $\alpha$ and $\beta$ are even integers and $p$ is an odd prime. Also $p=[F(\alpha, \beta)]$ in which $F(\alpha, \beta)=2^{\alpha+1} \cdot 3^{\beta+1} /\left(2^{\alpha+1}+3^{\beta+1}-1\right)$ (Here $[x]$, as usual, denotes the greatest integer not exceeding the real number $x$ ).

Lemma 1.2 ([3, Theorem 1.1]). If $n$ is $Q M$ with $\omega(n)=4$, then $n$ is $Q T$ and is of the form $n=2^{\alpha} \cdot 3^{\beta} \cdot p^{\gamma} \cdot q^{\delta}$, where $\alpha, \beta, \gamma$ and $\delta$ are even integers and $p<q$ are odd primes.

The purpose of this paper is to give some necessary conditions on the prime $p$ in Lemma 1.1 and on the primes $p$ and $q$ in Lemma 1.2. Also we establish that no QM number with $\omega(n)=4$ can be a fourth power of an integer In fact, we prove the following:

Theorem A. The odd prime $p$ in Lemma 1.1 is such that

$$
\begin{aligned}
(i) p & \equiv 29(\bmod 36) \text { if } \alpha \equiv 0(\bmod 6) \\
(\text { ii } p & \equiv 17(\bmod 36) \text { if } \alpha \equiv 2(\bmod 6) \\
\text { and }(\text { iii } p & \equiv 5(\bmod 36) \text { if } \alpha \equiv 4(\bmod 6) .
\end{aligned}
$$

Theorem B. Suppose $n$ is QM with $\omega(n)=4$ and is of the form given in Lemma 1.2. Suppose $p \equiv a(\bmod 8)$ and $q \equiv b(\bmod 8)$. Then

$$
\begin{aligned}
(i)(a, b) & \notin\{(3,3),(3,7),(7,3),(7,7)\} \\
(i i)(a, b) & \in\{(1,3),(5,3),(1,7),(5,7)\} \text { implies } \gamma \equiv 2(\bmod 4) \\
(\text { iii })(a, b) & \in\{(3,1),(3,5),(7,1),(7,5)\} \text { implies } \delta \equiv 2(\bmod 4) \\
\text { and }(\text { iv })(a, b) & \in\{(1,1),(1,5),(5,1),(5,5)\} \text { implies } \gamma \equiv 2(\bmod 4) \text { or } \delta \equiv 2(\bmod 4) .
\end{aligned}
$$

Remark 1.3. In view of Theorem B, one of $p^{\gamma}$ and $q^{\delta}$ in Lemma 1.2 is not a fourth power and therefore the number $n$ in it cannot be a fourth power. That is, any QM $n$ with $\omega(n)=4$ is not a fourth power.

\section{On QM numbers $n$ with $\omega(n)=3$}

In this section $n$ always denotes a QM number with $\omega(n)=3$ so that, by Lemma $1.1 ., 3 n+1=$ $\sigma(n)$ and is of the form

$$
n=2^{\alpha} \cdot 3^{\beta} \cdot p^{2},
$$

where $\alpha$ and $\beta$ are even integers and $p$ is an odd prime given by $p=[F(\alpha, \beta)]$. Therefore we have

$$
\begin{aligned}
3.2^{\alpha} \cdot 3^{\beta} \cdot p^{2}+1 & =\sigma\left(2^{\alpha}\right) \cdot \sigma\left(3^{\beta}\right) \cdot \sigma\left(p^{2}\right) \\
& =\left(2^{\alpha+1}-1\right) \cdot\left(\frac{3^{\beta+1}-1}{2}\right) \cdot\left(1+p+p^{2}\right),
\end{aligned}
$$

which can be written as

$$
2^{\alpha+1} \cdot 3^{\beta+1} \cdot p^{2}+2=\left(2^{\alpha+1}-1\right) \cdot\left(3^{\beta+1}-1\right) \cdot\left(1+p+p^{2}\right) .
$$


First we prove

Lemma 2.1. $\alpha \geq 6$ and $\beta \geq 6$.

Proof. Note that $\alpha, \beta \in\{2,4,6,8, \ldots\}$ and that $F(2,2)=6.352, F(2,4)=7.776$, $F(4,2)=14.896$ and $F(4,4)=28.3785$. Therefore $[F(2,2)]=6,[F(2,4)]=7,[F(4,2)]=14$ and $[\mathrm{F}(4,4)]=28$ showing that for $(\alpha, \beta) \in\{(2,2),(4,2),(4,4)\}$ the values of $[F(\alpha, \beta)]$ are composite so that the corresponding numbers $n$ given in (2.1) are not QT. Also if $(\alpha, \beta)=(2,4)$, then $p=7$ so that in this case $n=2^{2} \cdot 3^{4} \cdot 7^{2}=15876$ for which $\sigma(n)=\sigma\left(2^{2}\right) \cdot \sigma\left(3^{4}\right) \cdot \sigma\left(7^{2}\right)$ $=7.121 .57=48279$ showing $3 n+1 \neq \sigma(n)$. Therefore it is not QT. Thus for QT of the form (2.1) we have $(\alpha, \beta) \notin\{(2,2),(2,4),(4,2),(4,4)\}$, proving the lemma.

Lemma 2.2. The prime $p$ in $(2.1)$ is such that $p \equiv 1(\bmod 4)$.

Proof. For the integers $\alpha$ and $\beta$, it is clear that $2^{\alpha+1}-1 \equiv-1(\bmod 8)$ and $3^{\beta+1}-1 \equiv 2(\bmod 8)$ so that

$$
\left(2^{\alpha+1}-1\right) \cdot\left(3^{\beta+1}-1\right) \equiv-2(\bmod 8) .
$$

Writing the equation (2.2) to congruence modulo 8 and using (2.3) we get

$$
2 \equiv-2\left(1+p+p^{2}\right)(\bmod 8) .
$$

That is, the prime $p$ should satisfy

$$
2+p+p^{2} \equiv 0(\bmod 4) .
$$

Now $p$, being an odd prime, we have $p \equiv 1$ or $3(\bmod 4)$ and in both cases $p^{2} \equiv 1(\bmod 4)$. Here (2.4) holds only if $p \equiv 1(\bmod 4)$, proving the lemma.

Lemma 2.3. The prime $p$ in $(2.1)$ is such that $p \equiv 2,8$ or $5(\bmod 9)$ according to $\alpha \equiv 0,2$ or $4(\bmod 6)$.

Proof. Write the equation (2.2) to congruence modulo 9 and use the fact that $3^{\beta+1}-1 \equiv$ $-1(\bmod 9)$ for $\beta \geq 1$ to get

$$
2+G(\alpha, p) \equiv 0(\bmod 9)
$$

where $G(\alpha, p)=\left(2^{\alpha+1}-1\right)\left(1+p+p^{2}\right)$.

Now $\alpha$, being an even integer, $\alpha \equiv 0,2$ or $4(\bmod 6)$ so that $\alpha=6 k, 6 k+2$ or $6 k+4$ for some integer $k \geq 1$ (in view of Lemma 2.1). Hence

$$
2^{\alpha+1}-1= \begin{cases}2\left(2^{6}\right)^{k}-1 & \text { if } \alpha \equiv 0(\bmod 6) \\ 2^{3}\left(2^{6}\right)^{k}-1 & \text { if } \alpha \equiv 2(\bmod 6) \\ 2^{5}\left(2^{6}\right)^{k}-1 & \text { if } \alpha \equiv 4(\bmod 6)\end{cases}
$$

so that

$$
2^{\alpha+1}-1 \equiv \begin{cases}1(\bmod 9) & \text { if } \alpha \equiv 0(\bmod 6) \\ 7(\bmod 9) & \text { if } \alpha \equiv 2(\bmod 6) \\ 4(\bmod 9) & \text { if } \alpha \equiv 4(\bmod 6)\end{cases}
$$

since $2^{6} \equiv 1(\bmod 9)$ 
For an odd prime $p$ we have $p \equiv 1,2,4,5,7$ or $8(\bmod 9)$ and in these respective cases $p^{2} \equiv 1,4,7,7,4$ or $1(\bmod 9)$. Therefore,

$$
1+p+p^{2} \equiv \begin{cases}3(\bmod 9) & \text { if } p \equiv 1(\bmod 9) \\ 7(\bmod 9) & \text { if } p \equiv 2(\bmod 9) \\ 3(\bmod 9) & \text { if } p \equiv 4(\bmod 9) \\ 4(\bmod 9) & \text { if } p \equiv 5(\bmod 9) \\ 3(\bmod 9) & \text { if } p \equiv 7(\bmod 9) \\ 1(\bmod 9) & \text { if } p \equiv 8(\bmod 9)\end{cases}
$$

For different cases of $\alpha \equiv 0,2$ or $4(\bmod 6)$ and for different cases of $p \equiv 1,2,4,5,7$ or $8(\bmod 9)$, the values of $k$ such that $G(\alpha, p) \equiv k(\bmod 9)$ are given in Table 1 below, using (2.6) and (2.7):

\begin{tabular}{|c||c|c|c|c|c|c|}
\hline & $p \equiv 1(\bmod 9)$ & $p \equiv 2(\bmod 9)$ & $p \equiv 4(\bmod 9)$ & $p \equiv 5(\bmod 9)$ & $p \equiv 7(\bmod 9)$ & $p \equiv 8(\bmod 9)$ \\
\hline \hline$\alpha \equiv 0(\bmod 6)$ & 3 & 7 & 3 & 4 & 3 & 1 \\
\hline$\alpha \equiv 2(\bmod 6)$ & 3 & 4 & 3 & 1 & 3 & 7 \\
\hline$\alpha \equiv 4(\bmod 6)$ & 3 & 1 & 3 & 7 & 3 & 4 \\
\hline
\end{tabular}

Table 1 . The values of $k$ such that $G(\alpha, p) \equiv k(\bmod 9)$.

It is clear from the Table 1 that $(2.5)$ holds only in the cases (i) $\alpha \equiv 0(\bmod 6), p \equiv 2(\bmod 9)$ (ii) $\alpha \equiv 2(\bmod 6), p \equiv 8(\bmod 9)$ and $($ iii) $\alpha \equiv 4(\bmod 6), p \equiv 5(\bmod 9)$, proving the lemma.

Proof of Theorem $A$. (i) Suppose $\alpha \equiv 0(\bmod 6)$, so that by Lemma 2.3, we have $p \equiv 2(\bmod 9)$. Also by Lemma $2.2, p \equiv 1(\bmod 4)$. Hence by the Chinese remainder theorem, we have $p \equiv 29(\bmod 36)$.

Parts (ii) and (iii) of Theorem A can be proved similarly, using Lemmas 2.2 and 2.3.

\section{On QM numbers $n$ with $\omega(n)=4$}

Throughout this section $n$ stands for a QM number with $\omega(n)=4$ so that, by Lemma 1.2, $\sigma(n)=3 n+1$ and $n$ is of the form

$$
n=2^{\alpha} \cdot 3^{\beta} \cdot p^{\gamma} \cdot q^{\delta},
$$

where $\alpha, \beta, \gamma$ and $\delta$ are even integers; and $p<q$ are odd primes. Therefore

$$
3.2^{\alpha} \cdot 3^{\beta} \cdot p^{\gamma} \cdot q^{\delta}+1=\left(2^{\alpha+1}-1\right)\left(\frac{3^{\beta+1}-1}{2}\right) \cdot \sigma\left(p^{\gamma}\right) \cdot \sigma\left(q^{\delta}\right)
$$

which can be written as

$$
2^{\alpha+1} \cdot 3^{\beta+1} \cdot p^{\gamma} \cdot q^{\delta}+2=\left(2^{\alpha+1}-1\right)\left(3^{\beta+1}-1\right) \cdot \sigma\left(p^{\gamma}\right) \cdot \sigma\left(q^{\delta}\right) .
$$


Proof of Theorem B. Writing the equation (3.2) to congruence modulo 8 and using (2.3) we get that $p, q, \gamma$ and $\delta$ of (3.1) must satisfy that $2 \equiv-2 . \sigma\left(p^{\gamma}\right) \sigma\left(q^{\delta}\right)(\bmod 8)$ or equivalently

$$
\sigma\left(p^{\gamma}\right) \sigma\left(q^{\delta}\right)+1 \equiv 0(\bmod 4)
$$

Now $p \equiv 1,3,5$ or $7(\bmod 8)$ so that $p^{2} \equiv 1(\bmod 8)$ in each case. Let $\gamma=2 a$ for some $a \geq 1$. Then

$$
\begin{aligned}
\sigma\left(p^{\gamma}\right) & =\sigma\left(p^{2 a}\right)=1+p+p^{2}+\ldots+p^{2 a} \\
& =(1+p)\left(1+p^{2}+\ldots+p^{2(a-1)}\right)+p^{2 a} \\
\equiv & (1+p) a+1(\bmod 8) \\
& \equiv \begin{cases}2 a+1(\bmod 8) & \text { if } p \equiv 1(\bmod 8) \\
4 a+1(\bmod 8) & \text { if } p \equiv 3(\bmod 8) \\
6 a+1(\bmod 8) & \text { if } p \equiv 5(\bmod 8) \\
1(\bmod 8) & \text { if } p \equiv 7(\bmod 8)\end{cases}
\end{aligned}
$$

which shows

Similarly

$$
\sigma\left(p^{\gamma}\right) \equiv \begin{cases}\gamma+1(\bmod 8) & \text { if } p \equiv 1(\bmod 8) \\ 2 \gamma+1(\bmod 8) & \text { if } p \equiv 3(\bmod 8) \\ 3 \gamma+1(\bmod 8) & \text { if } p \equiv 5(\bmod 8) \\ 1(\bmod 8) & \text { if } p \equiv 7(\bmod 8)\end{cases}
$$

$$
\sigma\left(q^{\delta}\right) \equiv \begin{cases}\delta+1(\bmod 8) & \text { if } q \equiv 1(\bmod 8) \\ 2 \delta+1(\bmod 8) & \text { if } q \equiv 3(\bmod 8) \\ 3 \delta+1(\bmod 8) & \text { if } q \equiv 5(\bmod 8) \\ 1(\bmod 8) & \text { if } q \equiv 7(\bmod 8)\end{cases}
$$

Table 2 gives the values of $k$ such that $\sigma\left(p^{\gamma}\right) \sigma\left(q^{\delta}\right) \equiv k(\bmod 8)$ for different cases of $p \equiv 1,3,5$ or $7(\bmod 8)$ and for different cases of $q \equiv 1,3,5$ or $7(\bmod 8)$.

\begin{tabular}{|l||c|c|c|c|}
\hline & $p \equiv 1(\bmod 8)$ & $p \equiv 3(\bmod 8)$ & $p \equiv 5(\bmod 8)$ & $p \equiv 7(\bmod 8)$ \\
\hline \hline$q \equiv 1(\bmod 8)$ & $(\gamma+1)(\delta+1)$ & $(2 \gamma+1)(\delta+1)$ & $(3 \gamma+1)(\delta+1)$ & $\delta+1$ \\
\hline$q \equiv 3(\bmod 8)$ & $(\gamma+1)(2 \delta+1)$ & $(2 \gamma+1)(2 \delta+1)$ & $(3 \gamma+1)(2 \delta+1)$ & $2 \delta+1$ \\
\hline$q \equiv 5(\bmod 8)$ & $(\gamma+1)(3 \delta+1)$ & $(2 \gamma+1)(3 \delta+1)$ & $(3 \gamma+1)(3 \delta+1)$ & $3 \delta+1$ \\
\hline$q \equiv 7(\bmod 8)$ & $(\gamma+1)$ & $(2 \gamma+1)$ & $(3 \gamma+1)$ & 1 \\
\hline
\end{tabular}

Table 2. The values of $k$ such that $\sigma\left(p^{\gamma}\right) \sigma\left(q^{\delta}\right) \equiv k(\bmod 8)$.

In view of (3.3), the values of $k$ must satisfy the condition

$$
k+1 \equiv 0(\bmod 4) .
$$

Now Theorem B follows from (3.6) and the Table 2. For instance, 
(i) if $(a, b)=(3,3)$, then $(2 \gamma+1)(2 \delta+1)+1 \equiv 0(\bmod 4)$ and this is impossible since $\gamma$ and $\delta$ are even integers, giving $(a, b) \neq(3,3)$.

(ii) if $(a, b)=(5,3)$, then $(3 \gamma+1)(2 \delta+1)+1 \equiv 0(\bmod 4)$ holds only for $(\gamma+1)+1 \equiv 0(\bmod 4)$ giving $\gamma \equiv 2(\bmod 4)$. That is, $(a, b)=(5,3) \Rightarrow \gamma \equiv 2(\bmod 4)$.

(iii) if $(a, b)=(3,1)$, then $(2 \gamma+1)(\delta+1)+1 \equiv 0(\bmod 4) \Rightarrow \delta+2 \equiv 0(\bmod 4)$ or $\delta \equiv$ $2(\bmod 4)$. That is, $(a, b)=(3,1) \Rightarrow \delta \equiv 2(\bmod 4)$.

(iv) if $(a, b)=(1,5)$, then $(\gamma+1)(3 \delta+1)+1 \equiv 0(\bmod 4) \Rightarrow \gamma+\delta+2 \equiv 0(\bmod 4)$ showing either $\gamma \equiv 2(\bmod 4)$ or $\delta \equiv 2(\bmod 4)$, but not both. That is, $(a, b)=(1,5) \Rightarrow \gamma \equiv$ $2(\bmod 4)$ or $\delta \equiv 2(\bmod 4)$.

The other cases can be proved similarly.

\section{Acknowledgements}

The authors are grateful to the referees for their valuable suggestions in improving the quality of the presentation.

\section{References}

[1] Abbott, H. L., Aull, C. E., Brown, E., \& Suryanarayana, D. (1973). Quasiperfect numbers, Acta Arithmetica, XXII, 439-447; Correction to the paper, Acta Arithmetica, XXIV (1976), 427-428.

[2] Cattaneo, P. (1951). Sui numeri quasiperfetti, Boll. Un. Mat. Ital., 6 (3), 59-62.

[3] Li, Meng, \& Min, Tang. (2014). On the congruence $\sigma(n) \equiv 1(\bmod n)-$ II, J. Math. Res. Appl., 34 (2), 155-160.

[4] Min, Tang \& Li, Meng. (2012). On the congruence $\sigma(n) \equiv 1(\bmod n)$, J. Math. Res. Appl., 32 (6) 673-676.

[5] Prasad, V. S. R., \& Sunitha, C. (2017). On quasiperfect numbers, Notes on Number Theory and Discrete Mathematics, 23 (3), 73-78.

[6] Prasad, V. S. R., \& Sunitha, C. (2019). On the prime factors of a quasiperfect number, Notes on Number Theory and Discrete Mathematics, 25 (2), 16-21.

[7] Sandor J., \& Crstici, B. (2004). Handbook of Number Theory II, Kluwer Academic Publishers, Dordrecht/ Boston/ London. 